\title{
Object-oriented programming semantics education based on intelligent agents
}

\begin{abstract}
Comprehending Object-Oriented Programming (OOP) concepts is a difficult task especially for novice students. This usually happens during the transition form learning fundamental concepts to object-oriented (OO) concepts. When given an OO problem to solve, novices find it hard to relate with objects. If novices can view the world based on real objects, this can help them solve their problem of comprehending the OO concepts. In this paper, we propose to design an agent model to understand the semantics of OO Java source codes. The agents are designed based on the Belief-Desire-Intention (BDI) architecture. Three agents namely GUI agent, semantic agent and novice agents are constructed. The GUI agent is controlled by the user to provide source codes examples. The semantic agent submits the source codes to the novice agent and explains the semantic or meaning of the source codes to the novice agent. The process involves source codes comparison technique. This model provides an OOP semantics knowledge representation based on intelligent agents.
\end{abstract}

Keyword: OOP; Intelligent agents; Source code comparison; Semantics; Knowledge representation 